\title{
Novel Structures and Pauson-Khand Activities of N-Heterocyclic Carbene Dicobalt Complexes
}

\author{
Susan E. Gibson, ${ }^{*} \dagger, \S$ Craig Johnstone, ${ }^{\ddagger}$ Jennifer A. Loch, $,{ }^{*} \dagger, \S$ Jonathan W. Steed, ${ }^{\dagger}$ and \\ Andrea Stevenazzi ${ }^{\dagger}$ \\ Department of Chemistry, King's College London, Strand, London \\ WC2R 2LS, United Kingdom, and AstraZeneca UK Ltd, Mereside, Alderley Park, \\ Macclesfield SK10 4TG, United Kingdom
}

The structures of 7 and 9 were solved and refined using the programs SHELXS-9 $7^{1}$ and SHELXL-97, ${ }^{2}$ respectively. The program X-Seed ${ }^{3}$ was used as an interface to the SHELX programs and to prepare the figures.

(1) Sheldrick, G. M. Acta Crystallogr., Sect. A 1990, 46, 467.

(2) Sheldrick, G. M. University of Göttingen, 1997.

(3) Barbour, L. J. J. Supramol. Chem. 2001, 1, 189. 
Table 1. Crystal data and structure refinement for 7 .

Identification code

Empirical formula

Formula weight

Temperature

Wavelength

Crystal system

Space group

Unit cell dimensions

Volume

Z

Density (calculated)

Absorption coefficient

$\mathrm{F}(000)$

Crystal size

Theta range for data collection

Index ranges

Reflections collected

Independent reflections

Completeness to theta $=27.48^{\circ}$

Absorption correction

Max. and min. transmission

Refinement method

Data / restraints / parameters

Goodness-of-fit on $\mathrm{F}^{2}$

Final R indices [I $>2 \operatorname{sigma}(\mathrm{I})]$

$\mathrm{R}$ indices (all data)

Extinction coefficient

Largest diff. peak and hole
C:.CIF

C45 H39 Co2 N2 O6 P

852.61

120(2) K

$0.71073 \AA$

Monoclinic

$\mathrm{P} 21 / \mathrm{c}$

$\mathrm{a}=10.3815(3) \AA$

$\alpha=90^{\circ}$.

$\mathrm{b}=23.4351(7) \AA$

$\beta=105.2150(10)^{\circ}$.

$\mathrm{c}=17.6562(6) \AA$

$\gamma=90^{\circ}$.

$4145.0(2) \AA^{3}$

4

$1.366 \mathrm{Mg} / \mathrm{m}^{3}$

$0.888 \mathrm{~mm}^{-1}$

1760

$0.40 \times 0.35 \times 0.20 \mathrm{~mm}^{3}$

1.48 to $27.48^{\circ}$.

$-13<=\mathrm{h}<=12,-27<=\mathrm{k}<=30,-19<=1<=20$

10402

$6805[\mathrm{R}(\mathrm{int})=0.0588]$

$71.6 \%$

Semi-empirical from equivalents

0.8423 and 0.7176

Full-matrix least-squares on $\mathrm{F}^{2}$

$6805 / 0 / 512$

1.076

$\mathrm{R} 1=0.0507, \mathrm{wR} 2=0.0813$

$\mathrm{R} 1=0.0869, \mathrm{wR} 2=0.0919$

$0.0007(2)$

0.448 and -0.413 e. $\AA^{-3}$ 
Table 2. Atomic coordinates ( $\left.\mathrm{x} 10^{4}\right)$ and equivalent isotropic displacement parameters $\left(\AA^{2} \times 10^{3}\right)$ for $7 . \mathrm{U}(\mathrm{eq})$ is defined as one third of the trace of the orthogonalized $\mathrm{U}^{\mathrm{ij}}$ tensor.

\begin{tabular}{|c|c|c|c|c|}
\hline & $\mathrm{x}$ & $\mathrm{y}$ & $\mathrm{z}$ & $\mathrm{U}(\mathrm{eq})$ \\
\hline $\operatorname{Co}(1)$ & 296(1) & 5394(1) & $2728(1)$ & $17(1)$ \\
\hline $\mathrm{P}(1)$ & $-3126(1)$ & $6682(1)$ & $1260(1)$ & $17(1)$ \\
\hline $\mathrm{O}(1)$ & $-1927(2)$ & $4577(1)$ & $2457(1)$ & $29(1)$ \\
\hline $\mathrm{N}(1)$ & 1946(2) & $4328(1)$ & $2848(1)$ & $17(1)$ \\
\hline$C(1)$ & $-1041(3)$ & $4888(1)$ & $2543(2)$ & $20(1)$ \\
\hline $\mathrm{Co}(2)$ & $-1544(1)$ & $6137(1)$ & 1951(1) & $18(1)$ \\
\hline $\mathrm{O}(2)$ & 297(2) & $6162(1)$ & $4053(1)$ & $30(1)$ \\
\hline $\mathrm{N}(2)$ & $2795(2)$ & $4914(1)$ & $3780(1)$ & $16(1)$ \\
\hline$C(2)$ & $351(3)$ & $5844(1)$ & $3560(2)$ & $21(1)$ \\
\hline $\mathrm{O}(3)$ & $1775(2)$ & $5871(1)$ & $1668(1)$ & $35(1)$ \\
\hline$C(3)$ & $1176(3)$ & $5670(1)$ & 2071(2) & $23(1)$ \\
\hline $\mathrm{O}(4)$ & $471(2)$ & $7034(1)$ & $2418(1)$ & $42(1)$ \\
\hline$C(4)$ & $-319(3)$ & $6683(1)$ & $2241(2)$ & $25(1)$ \\
\hline $\mathrm{O}(5)$ & $-1495(2)$ & $5385(1)$ & $635(1)$ & $31(1)$ \\
\hline$C(5)$ & $-1513(3)$ & $5680(1)$ & $1161(2)$ & $21(1)$ \\
\hline $\mathrm{O}(6)$ & $-3084(2)$ & $5832(1)$ & $3064(1)$ & $42(1)$ \\
\hline$C(6)$ & $-2461(3)$ & $5944(1)$ & $2631(2)$ & $25(1)$ \\
\hline$C(7)$ & 1706(3) & $4845(1)$ & $3152(2)$ & $16(1)$ \\
\hline$C(8)$ & $3138(3)$ & $4098(1)$ & $3267(2)$ & $23(1)$ \\
\hline$C(9)$ & $3676(3)$ & $4461(1)$ & $3852(2)$ & $23(1)$ \\
\hline$C(10)$ & 2943(3) & $5353(1)$ & $4366(2)$ & $16(1)$ \\
\hline $\mathrm{C}(11)$ & 3539(3) & $5871(1)$ & $4262(2)$ & $19(1)$ \\
\hline$C(12)$ & $3572(3)$ & $6295(1)$ & $4816(2)$ & $24(1)$ \\
\hline$C(13)$ & 3091(3) & $6210(1)$ & $5468(2)$ & $26(1)$ \\
\hline$C(14)$ & 2556(3) & $5682(1)$ & $5563(2)$ & $25(1)$ \\
\hline$C(15)$ & 2444(3) & $5248(1)$ & $5009(2)$ & $20(1)$ \\
\hline$C(16)$ & 4099(3) & $5973(1)$ & $3569(2)$ & $33(1)$ \\
\hline$C(17)$ & $3144(3)$ & $6678(1)$ & $6064(2)$ & $45(1)$ \\
\hline$C(18)$ & 1753(3) & $4692(1)$ & $5097(2)$ & $32(1)$ \\
\hline$C(19)$ & 1045(3) & $4035(1)$ & $2197(2)$ & 19(1) \\
\hline$C(20)$ & $180(3)$ & $3631(1)$ & $2374(2)$ & $24(1)$ \\
\hline
\end{tabular}




\begin{tabular}{|c|c|c|c|c|}
\hline$C(21)$ & $-698(3)$ & $3356(1)$ & $1739(2)$ & $31(1)$ \\
\hline $\mathrm{C}(22)$ & $-723(3)$ & $3474(1)$ & $960(2)$ & $33(1)$ \\
\hline$C(23)$ & $200(3)$ & $3865(1)$ & $828(2)$ & $34(1)$ \\
\hline$C(24)$ & 1113(3) & $4142(1)$ & $1440(2)$ & $25(1)$ \\
\hline$C(25)$ & $162(3)$ & $3500(1)$ & $3203(2)$ & $32(1)$ \\
\hline$C(26)$ & $-1718(3)$ & $3185(1)$ & 292(2) & $51(1)$ \\
\hline $\mathrm{C}(27)$ & 2132(3) & $4535(1)$ & $1248(2)$ & $35(1)$ \\
\hline$C(28)$ & $-4035(3)$ & $7062(1)$ & $1864(2)$ & $18(1)$ \\
\hline$C(29)$ & $-5366(3)$ & 6961(1) & $1832(2)$ & $24(1)$ \\
\hline $\mathrm{C}(30)$ & $-5961(3)$ & $7230(1)$ & $2360(2)$ & $29(1)$ \\
\hline $\mathrm{C}(31)$ & $-5229(4)$ & $7597(1)$ & $2914(2)$ & $34(1)$ \\
\hline$C(32)$ & $-3912(4)$ & $7710(1)$ & $2945(2)$ & $35(1)$ \\
\hline $\mathrm{C}(33)$ & $-3316(3)$ & $7438(1)$ & $2428(2)$ & $27(1)$ \\
\hline $\mathrm{C}(34)$ & $-4384(3)$ & $6296(1)$ & $523(2)$ & $17(1)$ \\
\hline$C(35)$ & $-5062(3)$ & $5838(1)$ & $752(2)$ & $25(1)$ \\
\hline$C(36)$ & $-5976(3)$ & $5525(1)$ & $215(2)$ & $31(1)$ \\
\hline$C(37)$ & $-6216(3)$ & $5642(1)$ & $-578(2)$ & $29(1)$ \\
\hline $\mathrm{C}(38)$ & $-5531(3)$ & $6080(1)$ & $-816(2)$ & $27(1)$ \\
\hline $\mathrm{C}(39)$ & $-4628(3)$ & $6406(1)$ & $-274(2)$ & $24(1)$ \\
\hline $\mathrm{C}(40)$ & $-2630(3)$ & $7254(1)$ & $686(2)$ & $16(1)$ \\
\hline $\mathrm{C}(41)$ & $-1526(3)$ & $7182(1)$ & $396(2)$ & $25(1)$ \\
\hline $\mathrm{C}(42)$ & $-1215(3)$ & $7592(1)$ & $-96(2)$ & $32(1)$ \\
\hline $\mathrm{C}(43)$ & $-2000(3)$ & $8072(1)$ & $-300(2)$ & $26(1)$ \\
\hline $\mathrm{C}(44)$ & $-3098(3)$ & $8148(1)$ & $-10(2)$ & $23(1)$ \\
\hline$C(45)$ & $-3421(3)$ & $7738(1)$ & $478(2)$ & $19(1)$ \\
\hline
\end{tabular}


Table 3. Bond lengths $[\AA]$ and angles $\left[{ }^{\circ}\right]$ for 7.

\begin{tabular}{|c|c|}
\hline $\mathrm{Co}(1)-\mathrm{C}(3)$ & $1.778(4)$ \\
\hline $\mathrm{Co}(1)-\mathrm{C}(1)$ & $1.789(3)$ \\
\hline $\mathrm{Co}(1)-\mathrm{C}(2)$ & $1.798(4)$ \\
\hline $\mathrm{Co}(1)-\mathrm{C}(7)$ & $1.944(3)$ \\
\hline $\mathrm{Co}(1)-\mathrm{Co}(2)$ & $2.6819(5)$ \\
\hline $\mathrm{P}(1)-\mathrm{C}(34)$ & $1.824(3)$ \\
\hline $\mathrm{P}(1)-\mathrm{C}(28)$ & $1.831(3)$ \\
\hline $\mathrm{P}(1)-\mathrm{C}(40)$ & $1.834(3)$ \\
\hline $\mathrm{P}(1)-\mathrm{Co}(2)$ & $2.1832(9)$ \\
\hline $\mathrm{O}(1)-\mathrm{C}(1)$ & $1.152(3)$ \\
\hline $\mathrm{N}(1)-\mathrm{C}(7)$ & $1.373(3)$ \\
\hline $\mathrm{N}(1)-\mathrm{C}(8)$ & $1.374(4)$ \\
\hline $\mathrm{N}(1)-\mathrm{C}(19)$ & $1.451(4)$ \\
\hline $\mathrm{Co}(2)-\mathrm{C}(5)$ & $1.766(3)$ \\
\hline $\mathrm{Co}(2)-\mathrm{C}(6)$ & $1.776(4)$ \\
\hline $\mathrm{Co}(2)-\mathrm{C}(4)$ & $1.782(3)$ \\
\hline $\mathrm{O}(2)-\mathrm{C}(2)$ & $1.158(3)$ \\
\hline $\mathrm{N}(2)-\mathrm{C}(7)$ & $1.369(4)$ \\
\hline $\mathrm{N}(2)-\mathrm{C}(9)$ & $1.386(3)$ \\
\hline $\mathrm{N}(2)-\mathrm{C}(10)$ & $1.440(3)$ \\
\hline $\mathrm{O}(3)-\mathrm{C}(3)$ & $1.160(3)$ \\
\hline $\mathrm{O}(4)-\mathrm{C}(4)$ & $1.146(3)$ \\
\hline $\mathrm{O}(5)-\mathrm{C}(5)$ & $1.161(3)$ \\
\hline $\mathrm{O}(6)-\mathrm{C}(6)$ & $1.153(3)$ \\
\hline $\mathrm{C}(8)-\mathrm{C}(9)$ & $1.341(4)$ \\
\hline$C(10)-C(15)$ & $1.389(4)$ \\
\hline$C(10)-C(11)$ & $1.396(4)$ \\
\hline $\mathrm{C}(11)-\mathrm{C}(12)$ & $1.388(4)$ \\
\hline$C(11)-C(16)$ & $1.504(4)$ \\
\hline $\mathrm{C}(12)-\mathrm{C}(13)$ & $1.384(4)$ \\
\hline$C(13)-C(14)$ & $1.384(4)$ \\
\hline $\mathrm{C}(13)-\mathrm{C}(17)$ & $1.511(4)$ \\
\hline$C(14)-C(15)$ & $1.394(4)$ \\
\hline$C(15)-C(18)$ & $1.514(4)$ \\
\hline
\end{tabular}




\begin{tabular}{|c|c|}
\hline$C(19)-C(24)$ & $1.380(4)$ \\
\hline$C(19)-C(20)$ & $1.395(4)$ \\
\hline$C(20)-C(21)$ & $1.402(4)$ \\
\hline$C(20)-C(25)$ & $1.501(4)$ \\
\hline$C(21)-C(22)$ & $1.396(4)$ \\
\hline$C(22)-C(23)$ & $1.390(4)$ \\
\hline$C(22)-C(26)$ & $1.509(4)$ \\
\hline$C(23)-C(24)$ & $1.395(4)$ \\
\hline$C(24)-C(27)$ & $1.508(4)$ \\
\hline$C(28)-C(29)$ & $1.388(4)$ \\
\hline$C(28)-C(33)$ & $1.392(4)$ \\
\hline$C(29)-C(30)$ & $1.396(4)$ \\
\hline$C(30)-C(31)$ & $1.373(4)$ \\
\hline$C(31)-C(32)$ & $1.380(4)$ \\
\hline$C(32)-C(33)$ & $1.385(4)$ \\
\hline$C(34)-C(39)$ & $1.387(4)$ \\
\hline$C(34)-C(35)$ & $1.399(4)$ \\
\hline$C(35)-C(36)$ & $1.366(4)$ \\
\hline$C(36)-C(37)$ & $1.384(4)$ \\
\hline $\mathrm{C}(37)-\mathrm{C}(38)$ & $1.377(4)$ \\
\hline $\mathrm{C}(38)-\mathrm{C}(39)$ & $1.382(4)$ \\
\hline$C(40)-C(41)$ & $1.383(4)$ \\
\hline$C(40)-C(45)$ & $1.393(4)$ \\
\hline$C(41)-C(42)$ & $1.389(4)$ \\
\hline$C(42)-C(43)$ & $1.379(4)$ \\
\hline$C(43)-C(44)$ & $1.379(4)$ \\
\hline$C(44)-C(45)$ & $1.388(4)$ \\
\hline $\mathrm{C}(3)-\mathrm{Co}(1)-\mathrm{C}(1)$ & $128.99(14)$ \\
\hline $\mathrm{C}(3)-\mathrm{Co}(1)-\mathrm{C}(2)$ & $114.07(13)$ \\
\hline $\mathrm{C}(1)-\mathrm{Co}(1)-\mathrm{C}(2)$ & $113.28(13)$ \\
\hline $\mathrm{C}(3)-\mathrm{Co}(1)-\mathrm{C}(7)$ & $91.64(12)$ \\
\hline $\mathrm{C}(1)-\mathrm{Co}(1)-\mathrm{C}(7)$ & $95.88(12)$ \\
\hline $\mathrm{C}(2)-\mathrm{Co}(1)-\mathrm{C}(7)$ & $102.41(13)$ \\
\hline $\mathrm{C}(3)-\mathrm{Co}(1)-\mathrm{Co}(2)$ & $81.26(10)$ \\
\hline $\mathrm{C}(1)-\mathrm{Co}(1)-\mathrm{Co}(2)$ & $85.51(9)$ \\
\hline
\end{tabular}




\begin{tabular}{|c|c|}
\hline $\mathrm{C}(2)-\mathrm{Co}(1)-\mathrm{Co}(2)$ & $84.37(9)$ \\
\hline $\mathrm{C}(7)-\operatorname{Co}(1)-\operatorname{Co}(2)$ & $171.78(9)$ \\
\hline$C(34)-P(1)-C(28)$ & $105.99(14)$ \\
\hline$C(34)-P(1)-C(40)$ & $102.75(14)$ \\
\hline$C(28)-P(1)-C(40)$ & $103.24(13)$ \\
\hline $\mathrm{C}(34)-\mathrm{P}(1)-\mathrm{Co}(2)$ & 113.66(9) \\
\hline $\mathrm{C}(28)-\mathrm{P}(1)-\mathrm{Co}(2)$ & $112.70(10)$ \\
\hline $\mathrm{C}(40)-\mathrm{P}(1)-\mathrm{Co}(2)$ & $117.23(10)$ \\
\hline $\mathrm{C}(7)-\mathrm{N}(1)-\mathrm{C}(8)$ & $111.5(2)$ \\
\hline$C(7)-N(1)-C(19)$ & $125.6(2)$ \\
\hline $\mathrm{C}(8)-\mathrm{N}(1)-\mathrm{C}(19)$ & $122.8(2)$ \\
\hline $\mathrm{O}(1)-\mathrm{C}(1)-\mathrm{Co}(1)$ & $176.2(3)$ \\
\hline $\mathrm{C}(5)-\mathrm{Co}(2)-\mathrm{C}(6)$ & $120.29(14)$ \\
\hline $\mathrm{C}(5)-\mathrm{Co}(2)-\mathrm{C}(4)$ & $120.17(14)$ \\
\hline $\mathrm{C}(6)-\mathrm{Co}(2)-\mathrm{C}(4)$ & $117.22(15)$ \\
\hline $\mathrm{C}(5)-\mathrm{Co}(2)-\mathrm{P}(1)$ & $94.58(10)$ \\
\hline $\mathrm{C}(6)-\mathrm{Co}(2)-\mathrm{P}(1)$ & $93.98(10)$ \\
\hline $\mathrm{C}(4)-\mathrm{Co}(2)-\mathrm{P}(1)$ & $96.67(9)$ \\
\hline $\mathrm{C}(5)-\mathrm{Co}(2)-\mathrm{Co}(1)$ & $81.50(10)$ \\
\hline $\mathrm{C}(6)-\mathrm{Co}(2)-\mathrm{Co}(1)$ & $85.69(10)$ \\
\hline $\mathrm{C}(4)-\mathrm{Co}(2)-\mathrm{Co}(1)$ & $87.72(9)$ \\
\hline $\mathrm{P}(1)-\mathrm{Co}(2)-\mathrm{Co}(1)$ & $175.20(3)$ \\
\hline $\mathrm{C}(7)-\mathrm{N}(2)-\mathrm{C}(9)$ & $111.7(2)$ \\
\hline$C(7)-N(2)-C(10)$ & $125.2(2)$ \\
\hline $\mathrm{C}(9)-\mathrm{N}(2)-\mathrm{C}(10)$ & $122.6(3)$ \\
\hline $\mathrm{O}(2)-\mathrm{C}(2)-\mathrm{Co}(1)$ & $174.0(3)$ \\
\hline $\mathrm{O}(3)-\mathrm{C}(3)-\mathrm{Co}(1)$ & $176.6(3)$ \\
\hline $\mathrm{O}(4)-\mathrm{C}(4)-\mathrm{Co}(2)$ & 179.1(3) \\
\hline $\mathrm{O}(5)-\mathrm{C}(5)-\mathrm{Co}(2)$ & 179.2(3) \\
\hline $\mathrm{O}(6)-\mathrm{C}(6)-\mathrm{Co}(2)$ & $177.9(3)$ \\
\hline $\mathrm{N}(2)-\mathrm{C}(7)-\mathrm{N}(1)$ & $102.9(2)$ \\
\hline $\mathrm{N}(2)-\mathrm{C}(7)-\mathrm{Co}(1)$ & $127.7(2)$ \\
\hline $\mathrm{N}(1)-\mathrm{C}(7)-\mathrm{Co}(1)$ & $128.9(2)$ \\
\hline $\mathrm{C}(9)-\mathrm{C}(8)-\mathrm{N}(1)$ & $107.4(3)$ \\
\hline $\mathrm{C}(8)-\mathrm{C}(9)-\mathrm{N}(2)$ & $106.4(3)$ \\
\hline$C(15)-C(10)-C(11)$ & $122.4(3)$ \\
\hline
\end{tabular}




\begin{tabular}{|c|c|}
\hline$C(15)-C(10)-N(2)$ & $117.9(3)$ \\
\hline $\mathrm{C}(11)-\mathrm{C}(10)-\mathrm{N}(2)$ & $119.6(3)$ \\
\hline$C(12)-C(11)-C(10)$ & $117.3(3)$ \\
\hline $\mathrm{C}(12)-\mathrm{C}(11)-\mathrm{C}(16)$ & 121.1(3) \\
\hline$C(10)-C(11)-C(16)$ & $121.6(3)$ \\
\hline $\mathrm{C}(13)-\mathrm{C}(12)-\mathrm{C}(11)$ & $122.4(3)$ \\
\hline $\mathrm{C}(12)-\mathrm{C}(13)-\mathrm{C}(14)$ & $118.2(3)$ \\
\hline $\mathrm{C}(12)-\mathrm{C}(13)-\mathrm{C}(17)$ & $121.3(3)$ \\
\hline $\mathrm{C}(14)-\mathrm{C}(13)-\mathrm{C}(17)$ & $120.5(3)$ \\
\hline $\mathrm{C}(13)-\mathrm{C}(14)-\mathrm{C}(15)$ & $122.0(3)$ \\
\hline$C(10)-C(15)-C(14)$ & $117.5(3)$ \\
\hline $\mathrm{C}(10)-\mathrm{C}(15)-\mathrm{C}(18)$ & $121.6(3)$ \\
\hline $\mathrm{C}(14)-\mathrm{C}(15)-\mathrm{C}(18)$ & $120.8(3)$ \\
\hline$C(24)-C(19)-C(20)$ & $122.6(3)$ \\
\hline $\mathrm{C}(24)-\mathrm{C}(19)-\mathrm{N}(1)$ & 119.7(3) \\
\hline $\mathrm{C}(20)-\mathrm{C}(19)-\mathrm{N}(1)$ & $117.6(3)$ \\
\hline $\mathrm{C}(19)-\mathrm{C}(20)-\mathrm{C}(21)$ & $117.0(3)$ \\
\hline$C(19)-C(20)-C(25)$ & $122.0(3)$ \\
\hline $\mathrm{C}(21)-\mathrm{C}(20)-\mathrm{C}(25)$ & $121.0(3)$ \\
\hline $\mathrm{C}(22)-\mathrm{C}(21)-\mathrm{C}(20)$ & $122.5(3)$ \\
\hline $\mathrm{C}(23)-\mathrm{C}(22)-\mathrm{C}(21)$ & $117.4(3)$ \\
\hline $\mathrm{C}(23)-\mathrm{C}(22)-\mathrm{C}(26)$ & $121.8(4)$ \\
\hline $\mathrm{C}(21)-\mathrm{C}(22)-\mathrm{C}(26)$ & $120.8(3)$ \\
\hline $\mathrm{C}(22)-\mathrm{C}(23)-\mathrm{C}(24)$ & $122.3(3)$ \\
\hline$C(19)-C(24)-C(23)$ & $118.0(3)$ \\
\hline $\mathrm{C}(19)-\mathrm{C}(24)-\mathrm{C}(27)$ & 123.1(3) \\
\hline $\mathrm{C}(23)-\mathrm{C}(24)-\mathrm{C}(27)$ & $118.9(3)$ \\
\hline $\mathrm{C}(29)-\mathrm{C}(28)-\mathrm{C}(33)$ & $118.6(3)$ \\
\hline $\mathrm{C}(29)-\mathrm{C}(28)-\mathrm{P}(1)$ & $123.6(2)$ \\
\hline $\mathrm{C}(33)-\mathrm{C}(28)-\mathrm{P}(1)$ & $117.6(2)$ \\
\hline $\mathrm{C}(28)-\mathrm{C}(29)-\mathrm{C}(30)$ & $120.4(3)$ \\
\hline $\mathrm{C}(31)-\mathrm{C}(30)-\mathrm{C}(29)$ & $120.0(3)$ \\
\hline $\mathrm{C}(30)-\mathrm{C}(31)-\mathrm{C}(32)$ & $120.4(3)$ \\
\hline $\mathrm{C}(31)-\mathrm{C}(32)-\mathrm{C}(33)$ & $119.7(3)$ \\
\hline $\mathrm{C}(32)-\mathrm{C}(33)-\mathrm{C}(28)$ & $120.9(3)$ \\
\hline$C(39)-C(34)-C(35)$ & $117.7(3)$ \\
\hline
\end{tabular}




$\begin{array}{ll}\mathrm{C}(39)-\mathrm{C}(34)-\mathrm{P}(1) & 122.5(2) \\ \mathrm{C}(35)-\mathrm{C}(34)-\mathrm{P}(1) & 119.6(2) \\ \mathrm{C}(36)-\mathrm{C}(35)-\mathrm{C}(34) & 121.5(3) \\ \mathrm{C}(35)-\mathrm{C}(36)-\mathrm{C}(37) & 120.3(3) \\ \mathrm{C}(38)-\mathrm{C}(37)-\mathrm{C}(36) & 119.1(3) \\ \mathrm{C}(37)-\mathrm{C}(38)-\mathrm{C}(39) & 120.9(3) \\ \mathrm{C}(38)-\mathrm{C}(39)-\mathrm{C}(34) & 120.6(3) \\ \mathrm{C}(41)-\mathrm{C}(40)-\mathrm{C}(45) & 119.3(3) \\ \mathrm{C}(41)-\mathrm{C}(40)-\mathrm{P}(1) & 119.8(2) \\ \mathrm{C}(45)-\mathrm{C}(40)-\mathrm{P}(1) & 120.7(2) \\ \mathrm{C}(40)-\mathrm{C}(41)-\mathrm{C}(42) & 119.9(3) \\ \mathrm{C}(43)-\mathrm{C}(42)-\mathrm{C}(41) & 120.6(3) \\ \mathrm{C}(44)-\mathrm{C}(43)-\mathrm{C}(42) & 119.8(3) \\ \mathrm{C}(43)-\mathrm{C}(44)-\mathrm{C}(45) & 120.0(3) \\ \mathrm{C}(44)-\mathrm{C}(45)-\mathrm{C}(40) & 120.3(3)\end{array}$

Symmetry transformations used to generate equivalent atoms: 
Table 4. Anisotropic displacement parameters $\left(\AA^{2} \times 10^{3}\right)$ for 7 . The anisotropic displacement factor exponent takes the form: $-2 \pi^{2}\left[h^{2} a^{* 2} U^{11}+\ldots+2 h k a^{*} b^{*} U^{12}\right]$

\begin{tabular}{|c|c|c|c|c|c|c|}
\hline & $\mathrm{U}^{11}$ & $\mathrm{U}^{22}$ & $\mathrm{U}^{33}$ & $\mathrm{U}^{23}$ & $\mathrm{U}^{13}$ & $\mathrm{U}^{12}$ \\
\hline $\operatorname{Co}(1)$ & $17(1)$ & $17(1)$ & $15(1)$ & $1(1)$ & $1(1)$ & $3(1)$ \\
\hline $\mathrm{P}(1)$ & $17(1)$ & $17(1)$ & $16(1)$ & $2(1)$ & $3(1)$ & 1(1) \\
\hline $\mathrm{O}(1)$ & $23(2)$ & $28(1)$ & $33(2)$ & $2(1)$ & $4(1)$ & $-4(1)$ \\
\hline $\mathrm{N}(1)$ & $17(2)$ & $19(1)$ & $15(2)$ & $2(1)$ & $4(1)$ & $4(1)$ \\
\hline $\mathrm{C}(1)$ & $24(2)$ & $23(2)$ & $13(2)$ & $3(2)$ & $5(2)$ & $6(2)$ \\
\hline $\mathrm{Co}(2)$ & $17(1)$ & $18(1)$ & $16(1)$ & $2(1)$ & $1(1)$ & $2(1)$ \\
\hline $\mathrm{O}(2)$ & $27(2)$ & $40(1)$ & $22(2)$ & $-8(1)$ & $2(1)$ & $10(1)$ \\
\hline $\mathrm{N}(2)$ & $17(2)$ & $18(1)$ & $11(2)$ & $0(1)$ & $2(1)$ & $3(1)$ \\
\hline $\mathrm{C}(2)$ & $11(2)$ & $29(2)$ & $20(2)$ & $9(2)$ & $0(2)$ & $4(2)$ \\
\hline $\mathrm{O}(3)$ & $35(2)$ & $41(1)$ & $32(2)$ & $10(1)$ & $17(1)$ & $4(1)$ \\
\hline $\mathrm{C}(3)$ & $26(2)$ & $20(2)$ & $19(2)$ & $2(2)$ & $-2(2)$ & $7(2)$ \\
\hline $\mathrm{O}(4)$ & $30(2)$ & $25(1)$ & $62(2)$ & $-5(1)$ & $-6(1)$ & $-7(1)$ \\
\hline$C(4)$ & $24(2)$ & $18(2)$ & $27(2)$ & $4(2)$ & $-4(2)$ & $10(2)$ \\
\hline $\mathrm{O}(5)$ & $40(2)$ & $34(1)$ & $18(2)$ & $-3(1)$ & $4(1)$ & $0(1)$ \\
\hline $\mathrm{C}(5)$ & $18(2)$ & $24(2)$ & $19(2)$ & $8(2)$ & $0(2)$ & $2(2)$ \\
\hline $\mathrm{O}(6)$ & $36(2)$ & $60(2)$ & $35(2)$ & $19(1)$ & $20(1)$ & $18(1)$ \\
\hline$C(6)$ & $23(2)$ & $29(2)$ & $19(2)$ & $4(2)$ & $-3(2)$ & $10(2)$ \\
\hline$C(7)$ & $18(2)$ & $16(2)$ & $16(2)$ & $2(1)$ & $8(2)$ & $-1(1)$ \\
\hline$C(8)$ & $24(2)$ & $21(2)$ & $26(2)$ & $1(2)$ & $7(2)$ & $8(2)$ \\
\hline$C(9)$ & $17(2)$ & $27(2)$ & $23(2)$ & $4(2)$ & $2(2)$ & $9(2)$ \\
\hline$C(10)$ & $12(2)$ & $23(2)$ & $10(2)$ & $-3(2)$ & $-2(2)$ & $5(1)$ \\
\hline $\mathrm{C}(11)$ & $11(2)$ & $26(2)$ & $18(2)$ & $0(2)$ & $0(2)$ & $1(1)$ \\
\hline$C(12)$ & $15(2)$ & $22(2)$ & $30(2)$ & $-3(2)$ & $0(2)$ & $-2(1)$ \\
\hline$C(13)$ & $14(2)$ & $35(2)$ & $25(2)$ & $-14(2)$ & $-1(2)$ & $2(2)$ \\
\hline$C(14)$ & $23(2)$ & $40(2)$ & $12(2)$ & $0(2)$ & $3(2)$ & $4(2)$ \\
\hline$C(15)$ & $17(2)$ & $25(2)$ & $16(2)$ & $1(2)$ & $0(2)$ & $6(1)$ \\
\hline$C(16)$ & $32(2)$ & $37(2)$ & $29(2)$ & $0(2)$ & $8(2)$ & $-13(2)$ \\
\hline$C(17)$ & $41(3)$ & $49(2)$ & $44(3)$ & $-23(2)$ & $10(2)$ & $-9(2)$ \\
\hline$C(18)$ & $37(2)$ & $31(2)$ & $29(2)$ & $5(2)$ & $12(2)$ & $2(2)$ \\
\hline$C(19)$ & $18(2)$ & $20(2)$ & $16(2)$ & $-5(2)$ & $1(2)$ & $6(1)$ \\
\hline $\mathrm{C}(20)$ & $23(2)$ & $16(2)$ & $33(3)$ & $2(2)$ & $6(2)$ & $6(2)$ \\
\hline
\end{tabular}




\begin{tabular}{|c|c|c|c|c|c|c|}
\hline$C(21)$ & $16(2)$ & $20(2)$ & $56(3)$ & $-7(2)$ & $7(2)$ & $1(2)$ \\
\hline$C(22)$ & $28(2)$ & $32(2)$ & $32(3)$ & $-16(2)$ & $-3(2)$ & $12(2)$ \\
\hline$C(23)$ & $36(3)$ & $39(2)$ & $27(2)$ & $-11(2)$ & $7(2)$ & $5(2)$ \\
\hline$C(24)$ & $26(2)$ & $27(2)$ & $20(2)$ & $-6(2)$ & $4(2)$ & $7(2)$ \\
\hline$C(25)$ & $28(2)$ & $25(2)$ & $40(3)$ & $6(2)$ & $5(2)$ & 1(2) \\
\hline$C(26)$ & $31(2)$ & $56(2)$ & $57(3)$ & $-30(2)$ & $-5(2)$ & $8(2)$ \\
\hline$C(27)$ & $45(3)$ & $43(2)$ & $21(2)$ & $-6(2)$ & $16(2)$ & $-5(2)$ \\
\hline$C(28)$ & $24(2)$ & $15(2)$ & $14(2)$ & $6(1)$ & $6(2)$ & $2(1)$ \\
\hline$C(29)$ & $28(2)$ & $24(2)$ & $21(2)$ & $3(2)$ & $9(2)$ & $3(2)$ \\
\hline$C(30)$ & $27(2)$ & $35(2)$ & $31(3)$ & $9(2)$ & $17(2)$ & $8(2)$ \\
\hline$C(31)$ & $53(3)$ & $33(2)$ & $23(2)$ & $6(2)$ & $20(2)$ & $15(2)$ \\
\hline$C(32)$ & $50(3)$ & $32(2)$ & $23(2)$ & $-6(2)$ & $6(2)$ & $8(2)$ \\
\hline$C(33)$ & $28(2)$ & $28(2)$ & $25(2)$ & $-2(2)$ & $8(2)$ & $5(2)$ \\
\hline$C(34)$ & $16(2)$ & $17(2)$ & $17(2)$ & $-1(2)$ & $3(2)$ & $6(1)$ \\
\hline$C(35)$ & $29(2)$ & $26(2)$ & $19(2)$ & $4(2)$ & $3(2)$ & $-2(2)$ \\
\hline$C(36)$ & $35(2)$ & $28(2)$ & $27(2)$ & $2(2)$ & $2(2)$ & $-13(2)$ \\
\hline$C(37)$ & $29(2)$ & $26(2)$ & $25(2)$ & $-4(2)$ & $-4(2)$ & $-4(2)$ \\
\hline$C(38)$ & $34(2)$ & $32(2)$ & $11(2)$ & $0(2)$ & $1(2)$ & $-1(2)$ \\
\hline$C(39)$ & $25(2)$ & $25(2)$ & $20(2)$ & $2(2)$ & $3(2)$ & $-2(2)$ \\
\hline$C(40)$ & $15(2)$ & $18(2)$ & $16(2)$ & $0(1)$ & $4(2)$ & $-1(1)$ \\
\hline $\mathrm{C}(41)$ & $25(2)$ & $18(2)$ & $32(2)$ & $6(2)$ & $9(2)$ & $7(2)$ \\
\hline$C(42)$ & $32(2)$ & $28(2)$ & $41(3)$ & $8(2)$ & $22(2)$ & $5(2)$ \\
\hline$C(43)$ & $31(2)$ & $22(2)$ & $25(2)$ & $5(2)$ & $11(2)$ & $-5(2)$ \\
\hline$C(44)$ & $26(2)$ & $21(2)$ & $21(2)$ & $3(2)$ & $4(2)$ & $5(2)$ \\
\hline$C(45)$ & $11(2)$ & $24(2)$ & $22(2)$ & 1(2) & $4(2)$ & 1(1) \\
\hline
\end{tabular}


Table 5. Hydrogen coordinates ( $\left.\mathrm{x} 10^{4}\right)$ and isotropic displacement parameters $\left(\AA^{2} \times 10^{3}\right)$ for 7 .

\begin{tabular}{|c|c|c|c|c|}
\hline & $\mathrm{x}$ & $\mathrm{y}$ & z & $\mathrm{U}(\mathrm{eq})$ \\
\hline $\mathrm{H}(8)$ & 3512 & 3746 & 3161 & 28 \\
\hline $\mathrm{H}(9)$ & 4499 & 4416 & 4239 & 28 \\
\hline $\mathrm{H}(12)$ & 3937 & 6656 & 4745 & 28 \\
\hline $\mathrm{H}(14)$ & 2256 & 5614 & 6018 & 30 \\
\hline $\mathrm{H}(16 \mathrm{~A})$ & 4676 & 5652 & 3516 & 49 \\
\hline $\mathrm{H}(16 \mathrm{~B})$ & 4621 & 6326 & 3650 & 49 \\
\hline $\mathrm{H}(16 \mathrm{C})$ & 3366 & 6005 & 3091 & 49 \\
\hline $\mathrm{H}(17 \mathrm{~A})$ & 2321 & 6904 & 5913 & 68 \\
\hline $\mathrm{H}(17 \mathrm{~B})$ & 3912 & 6926 & 6082 & 68 \\
\hline $\mathrm{H}(17 \mathrm{C})$ & 3233 & 6509 & 6582 & 68 \\
\hline $\mathrm{H}(18 \mathrm{~A})$ & 929 & 4658 & 4674 & 47 \\
\hline $\mathrm{H}(18 \mathrm{~B})$ & 1538 & 4686 & 5605 & 47 \\
\hline $\mathrm{H}(18 \mathrm{C})$ & 2346 & 4372 & 5068 & 47 \\
\hline $\mathrm{H}(21)$ & -1299 & 3080 & 1842 & 37 \\
\hline $\mathrm{H}(23)$ & 211 & 3947 & 302 & 41 \\
\hline $\mathrm{H}(25 \mathrm{~A})$ & 1035 & 3351 & 3492 & 48 \\
\hline $\mathrm{H}(25 \mathrm{~B})$ & -527 & 3214 & 3202 & 48 \\
\hline $\mathrm{H}(25 \mathrm{C})$ & -33 & 3849 & 3457 & 48 \\
\hline $\mathrm{H}(26 \mathrm{~A})$ & -2570 & 3390 & 181 & 77 \\
\hline $\mathrm{H}(26 \mathrm{~B})$ & -1851 & 2790 & 438 & 77 \\
\hline $\mathrm{H}(26 \mathrm{C})$ & -1380 & 3187 & -176 & 77 \\
\hline $\mathrm{H}(27 \mathrm{~A})$ & 2698 & 4318 & 985 & 52 \\
\hline $\mathrm{H}(27 \mathrm{~B})$ & 2686 & 4702 & 1733 & 52 \\
\hline $\mathrm{H}(27 \mathrm{C})$ & 1674 & 4840 & 900 & 52 \\
\hline $\mathrm{H}(29)$ & -5874 & 6706 & 1450 & 29 \\
\hline $\mathrm{H}(30)$ & -6873 & 7159 & 2335 & 35 \\
\hline $\mathrm{H}(31)$ & -5631 & 7773 & 3279 & 41 \\
\hline $\mathrm{H}(32)$ & -3417 & 7974 & 3318 & 42 \\
\hline $\mathrm{H}(33)$ & -2402 & 7510 & 2459 & 32 \\
\hline $\mathrm{H}(35)$ & -4883 & 5744 & 1293 & 30 \\
\hline
\end{tabular}




\begin{tabular}{lrrrr}
$\mathrm{H}(36)$ & -6449 & 5226 & 387 & 37 \\
$\mathrm{H}(37)$ & -6844 & 5422 & -953 & 35 \\
$\mathrm{H}(38)$ & -5681 & 6159 & -1360 & 32 \\
$\mathrm{H}(39)$ & -4170 & 6710 & -449 & 29 \\
$\mathrm{H}(41)$ & -980 & 6853 & 534 & 30 \\
$\mathrm{H}(42)$ & -456 & 7542 & -294 & 38 \\
$\mathrm{H}(43)$ & -1784 & 8349 & -640 & 31 \\
$\mathrm{H}(44)$ & -3632 & 8480 & -143 & 28 \\
$\mathrm{H}(45)$ & -4186 & 7789 & 670 & 23 \\
\hline
\end{tabular}


Table 6. Crystal data and structure refinement for 9.

Identification code

Empirical formula

Formula weight

Temperature

Wavelength

Crystal system

Space group

Unit cell dimensions

Volume

Z

Density (calculated)

Absorption coefficient

$\mathrm{F}(000)$

Crystal size

Theta range for data collection

Index ranges

Reflections collected

Independent reflections

Completeness to theta $=25.00^{\circ}$

Absorption correction

Max. and min. transmission

Refinement method

Data / restraints / parameters

Goodness-of-fit on $\mathrm{F}^{2}$

Final $\mathrm{R}$ indices $[\mathrm{I}>2 \operatorname{sigma}(\mathrm{I})]$

$\mathrm{R}$ indices (all data)

Extinction coefficient

Largest diff. peak and hole
C:.CIF

C45 H41 Co2 N2 O6 P

854.63

120(2) K

$0.71073 \AA$

Triclinic

P-1

$\mathrm{a}=9.2002(4) \AA$

$\alpha=89.283(2)^{\circ}$.

$\mathrm{b}=12.6209(6) \AA$

$\beta=85.545(2)^{\circ}$.

$c=18.5026(10) \AA$

$\gamma=74.202(2)^{\circ}$.

2

$1.377 \mathrm{Mg} / \mathrm{m}^{3}$

$0.894 \mathrm{~mm}^{-1}$

884

$0.60 \times 0.30 \times 0.25 \mathrm{~mm}^{3}$

1.68 to $25.00^{\circ}$.

$-10<=\mathrm{h}<=10,-14<=\mathrm{k}<=15,-21<=\mathrm{l}<=21$

11979

$7191[\mathrm{R}($ int $)=0.1026]$

$99.4 \%$

Semi-empirical from equivalents

0.8075 and 0.6162

Full-matrix least-squares on $\mathrm{F}^{2}$

$7191 / 0 / 512$

1.136

$\mathrm{R} 1=0.0722, \mathrm{wR} 2=0.1324$

$\mathrm{R} 1=0.1266, \mathrm{wR} 2=0.1595$

$0.0032(8)$

0.656 and -1.107 e. $\AA^{-3}$ 
Table 7. Atomic coordinates ( $\left.\mathrm{x} 10^{4}\right)$ and equivalent isotropic displacement parameters $\left(\AA^{2} \times 10^{3}\right)$ for 9 . $U(e q)$ is defined as one third of the trace of the orthogonalized $U^{\mathrm{ij}}$ tensor.

\begin{tabular}{|c|c|c|c|c|}
\hline & $\mathrm{x}$ & $\mathrm{y}$ & z & $\mathrm{U}(\mathrm{eq})$ \\
\hline $\operatorname{Co}(1)$ & $5363(1)$ & 1937(1) & $2102(1)$ & $18(1)$ \\
\hline $\mathrm{P}(1)$ & $1177(2)$ & $5062(1)$ & $2916(1)$ & $19(1)$ \\
\hline $\mathrm{O}(1)$ & $3986(5)$ & $2319(3)$ & $700(2)$ & $36(1)$ \\
\hline $\mathrm{N}(1)$ & $8186(5)$ & $868(3)$ & $1218(2)$ & $22(1)$ \\
\hline$C(1)$ & $4588(6)$ & $2137(4)$ & $1225(3)$ & $23(1)$ \\
\hline $\mathrm{Co}(2)$ & 2931(1) & 3581(1) & $2546(1)$ & $20(1)$ \\
\hline $\mathrm{O}(2)$ & $3813(5)$ & $531(3)$ & $2970(2)$ & $35(1)$ \\
\hline $\mathrm{N}(2)$ & $8302(5)$ & $361(3)$ & $2343(2)$ & $20(1)$ \\
\hline$C(2)$ & $4486(6)$ & $1066(4)$ & $2650(3)$ & $22(1)$ \\
\hline $\mathrm{O}(3)$ & $6863(5)$ & $3497(3)$ & $2638(2)$ & $37(1)$ \\
\hline$C(3)$ & $6254(6)$ & $2868(4)$ & $2435(3)$ & $21(1)$ \\
\hline $\mathrm{O}(4)$ & $4121(5)$ & $4827(3)$ & $1410(2)$ & $38(1)$ \\
\hline$C(4)$ & $3668(7)$ & $4330(4)$ & $1854(3)$ & $24(1)$ \\
\hline $\mathrm{O}(5)$ & $966(5)$ & $2327(4)$ & $2032(2)$ & $43(1)$ \\
\hline$C(5)$ & $1745(7)$ & $2813(4)$ & $2244(3)$ & $24(1)$ \\
\hline $\mathrm{O}(6)$ & $4209(5)$ & $3133(3)$ & $3958(2)$ & $36(1)$ \\
\hline$C(6)$ & $3706(6)$ & $3286(4)$ & $3399(3)$ & $22(1)$ \\
\hline$C(7)$ & $7367(6)$ & $959(4)$ & $1863(3)$ & $16(1)$ \\
\hline$C(8)$ & $9824(7)$ & $-213(4)$ & $2014(3)$ & $29(2)$ \\
\hline$C(9)$ & $9777(6)$ & $224(4)$ & 1241(3) & $22(1)$ \\
\hline$C(10)$ & $7611(6)$ & $1218(4)$ & $522(3)$ & $21(1)$ \\
\hline $\mathrm{C}(11)$ & $7613(6)$ & $2269(4)$ & $263(3)$ & $21(1)$ \\
\hline$C(12)$ & $7090(6)$ & $2552(4)$ & $-411(3)$ & $25(1)$ \\
\hline$C(13)$ & $6634(6)$ & $1824(5)$ & $-836(3)$ & $26(1)$ \\
\hline$C(14)$ & $6669(6)$ & $782(4)$ & $-569(3)$ & $24(1)$ \\
\hline$C(15)$ & $7173(6)$ & $469(4)$ & $110(3)$ & $21(1)$ \\
\hline$C(16)$ & $8096(8)$ & $3079(5)$ & $706(3)$ & $38(2)$ \\
\hline$C(17)$ & $6080(7)$ & $2155(5)$ & $-1581(3)$ & $36(2)$ \\
\hline$C(18)$ & $7216(7)$ & $-674(4)$ & $385(3)$ & $34(2)$ \\
\hline$C(19)$ & $7909(6)$ & $90(4)$ & $3077(3)$ & $20(1)$ \\
\hline$C(20)$ & $7251(6)$ & $-793(4)$ & $3192(3)$ & $20(1)$ \\
\hline
\end{tabular}




\begin{tabular}{|c|c|c|c|c|}
\hline$C(21)$ & $6970(6)$ & $-1081(4)$ & $3905(3)$ & $25(1)$ \\
\hline$C(22)$ & 7306(7) & $-557(4)$ & $4498(3)$ & $25(1)$ \\
\hline$C(23)$ & 7967(7) & $299(5)$ & $4356(3)$ & $30(2)$ \\
\hline$C(24)$ & $8287(7)$ & $627(4)$ & $3657(3)$ & $26(1)$ \\
\hline$C(25)$ & $6917(7)$ & $-1426(4)$ & $2570(3)$ & $27(1)$ \\
\hline$C(26)$ & $6983(8)$ & $-915(5)$ & $5257(3)$ & $45(2)$ \\
\hline $\mathrm{C}(27)$ & $9065(8)$ & $1531(5)$ & $3539(4)$ & $43(2)$ \\
\hline $\mathrm{C}(28)$ & $-47(6)$ & $5666(4)$ & $2205(3)$ & $21(1)$ \\
\hline$C(29)$ & $-1365(6)$ & $5349(4)$ & $2109(3)$ & $25(1)$ \\
\hline $\mathrm{C}(30)$ & $-2204(7)$ & $5730(4)$ & 1531(3) & $30(1)$ \\
\hline $\mathrm{C}(31)$ & $-1764(7)$ & $6426(5)$ & $1026(3)$ & $31(2)$ \\
\hline$C(32)$ & $-447(7)$ & $6747(5)$ & 1115(3) & $27(1)$ \\
\hline $\mathrm{C}(33)$ & $407(6)$ & $6375(4)$ & $1698(3)$ & $22(1)$ \\
\hline $\mathrm{C}(34)$ & 1935(6) & $6188(4)$ & $3196(3)$ & $19(1)$ \\
\hline$C(35)$ & $3476(6)$ & $6018(4)$ & $3239(3)$ & $25(1)$ \\
\hline$C(36)$ & $4064(7)$ & $6856(4)$ & $3454(3)$ & $28(1)$ \\
\hline$C(37)$ & $3117(8)$ & $7888(5)$ & $3625(3)$ & $33(2)$ \\
\hline $\mathrm{C}(38)$ & $1589(8)$ & $8073(5)$ & $3585(3)$ & $35(2)$ \\
\hline $\mathrm{C}(39)$ & $986(7)$ & $7236(4)$ & $3370(3)$ & $29(1)$ \\
\hline $\mathrm{C}(40)$ & $-170(6)$ & 4921(4) & $3661(3)$ & $21(1)$ \\
\hline $\mathrm{C}(41)$ & $138(7)$ & $3967(4)$ & 4090(3) & $28(1)$ \\
\hline $\mathrm{C}(42)$ & $-860(7)$ & $3864(5)$ & $4676(3)$ & $35(2)$ \\
\hline $\mathrm{C}(43)$ & $-2150(7)$ & $4699(5)$ & $4850(3)$ & $34(2)$ \\
\hline $\mathrm{C}(44)$ & $-2460(7)$ & $5637(5)$ & $4443(3)$ & $37(2)$ \\
\hline$C(45)$ & $-1495(7)$ & $5747(5)$ & $3859(3)$ & $31(2)$ \\
\hline
\end{tabular}


Table 8. Bond lengths $[\AA]$ and angles $\left[{ }^{\circ}\right]$ for 9 .

\begin{tabular}{|c|c|}
\hline $\mathrm{Co}(1)-\mathrm{C}(3)$ & $1.747(6)$ \\
\hline $\mathrm{Co}(1)-\mathrm{C}(2)$ & $1.793(6)$ \\
\hline $\mathrm{Co}(1)-\mathrm{C}(1)$ & $1.811(6)$ \\
\hline $\mathrm{Co}(1)-\mathrm{C}(7)$ & $1.941(5)$ \\
\hline $\operatorname{Co}(1)-\operatorname{Co}(2)$ & $2.6856(9)$ \\
\hline $\mathrm{P}(1)-\mathrm{C}(28)$ & $1.821(5)$ \\
\hline $\mathrm{P}(1)-\mathrm{C}(40)$ & $1.822(5)$ \\
\hline $\mathrm{P}(1)-\mathrm{C}(34)$ & $1.840(6)$ \\
\hline $\mathrm{P}(1)-\mathrm{Co}(2)$ & $2.1876(15)$ \\
\hline $\mathrm{O}(1)-\mathrm{C}(1)$ & $1.145(6)$ \\
\hline $\mathrm{N}(1)-\mathrm{C}(7)$ & $1.349(6)$ \\
\hline $\mathrm{N}(1)-\mathrm{C}(10)$ & $1.448(7)$ \\
\hline $\mathrm{N}(1)-\mathrm{C}(9)$ & $1.472(6)$ \\
\hline $\mathrm{Co}(2)-\mathrm{C}(5)$ & $1.767(6)$ \\
\hline $\mathrm{Co}(2)-\mathrm{C}(6)$ & $1.776(6)$ \\
\hline $\mathrm{Co}(2)-\mathrm{C}(4)$ & $1.782(6)$ \\
\hline $\mathrm{O}(2)-\mathrm{C}(2)$ & $1.163(6)$ \\
\hline $\mathrm{N}(2)-\mathrm{C}(7)$ & $1.360(6)$ \\
\hline $\mathrm{N}(2)-\mathrm{C}(19)$ & $1.439(6)$ \\
\hline $\mathrm{N}(2)-\mathrm{C}(8)$ & $1.477(7)$ \\
\hline $\mathrm{O}(3)-\mathrm{C}(3)$ & $1.169(6)$ \\
\hline $\mathrm{O}(4)-\mathrm{C}(4)$ & $1.148(6)$ \\
\hline $\mathrm{O}(5)-\mathrm{C}(5)$ & $1.155(7)$ \\
\hline $\mathrm{O}(6)-\mathrm{C}(6)$ & $1.159(7)$ \\
\hline $\mathrm{C}(8)-\mathrm{C}(9)$ & $1.525(7)$ \\
\hline$C(10)-C(15)$ & $1.382(8)$ \\
\hline $\mathrm{C}(10)-\mathrm{C}(11)$ & $1.406(7)$ \\
\hline $\mathrm{C}(11)-\mathrm{C}(12)$ & $1.379(7)$ \\
\hline$C(11)-C(16)$ & $1.496(8)$ \\
\hline $\mathrm{C}(12)-\mathrm{C}(13)$ & $1.385(8)$ \\
\hline$C(13)-C(14)$ & $1.392(7)$ \\
\hline $\mathrm{C}(13)-\mathrm{C}(17)$ & $1.520(8)$ \\
\hline$C(14)-C(15)$ & $1.389(7)$ \\
\hline$C(15)-C(18)$ & $1.515(7)$ \\
\hline
\end{tabular}




\begin{tabular}{|c|c|}
\hline$C(19)-C(24)$ & $1.391(7)$ \\
\hline$C(19)-C(20)$ & $1.412(7)$ \\
\hline$C(20)-C(21)$ & $1.388(7)$ \\
\hline$C(20)-C(25)$ & $1.506(7)$ \\
\hline$C(21)-C(22)$ & $1.385(8)$ \\
\hline$C(22)-C(23)$ & $1.390(8)$ \\
\hline$C(22)-C(26)$ & $1.502(7)$ \\
\hline$C(23)-C(24)$ & $1.386(7)$ \\
\hline$C(24)-C(27)$ & $1.509(8)$ \\
\hline$C(28)-C(29)$ & $1.400(8)$ \\
\hline$C(28)-C(33)$ & $1.406(7)$ \\
\hline$C(29)-C(30)$ & $1.372(8)$ \\
\hline$C(30)-C(31)$ & $1.387(8)$ \\
\hline$C(31)-C(32)$ & $1.400(8)$ \\
\hline$C(32)-C(33)$ & $1.385(8)$ \\
\hline$C(34)-C(35)$ & $1.384(7)$ \\
\hline C(34)-C(39) & $1.398(7)$ \\
\hline$C(35)-C(36)$ & $1.387(8)$ \\
\hline$C(36)-C(37)$ & $1.380(8)$ \\
\hline $\mathrm{C}(37)-\mathrm{C}(38)$ & $1.368(9)$ \\
\hline C(38)-C(39) & $1.395(8)$ \\
\hline$C(40)-C(45)$ & $1.398(8)$ \\
\hline$C(40)-C(41)$ & $1.408(7)$ \\
\hline$C(41)-C(42)$ & $1.394(8)$ \\
\hline$C(42)-C(43)$ & $1.376(8)$ \\
\hline$C(43)-C(44)$ & $1.370(8)$ \\
\hline C(44)-C(45) & $1.376(8)$ \\
\hline $\mathrm{C}(3)-\mathrm{Co}(1)-\mathrm{C}(2)$ & $125.1(2)$ \\
\hline $\mathrm{C}(3)-\mathrm{Co}(1)-\mathrm{C}(1)$ & $120.8(2)$ \\
\hline $\mathrm{C}(2)-\mathrm{Co}(1)-\mathrm{C}(1)$ & 110.1(3) \\
\hline $\mathrm{C}(3)-\mathrm{Co}(1)-\mathrm{C}(7)$ & $87.5(2)$ \\
\hline $\mathrm{C}(2)-\mathrm{Co}(1)-\mathrm{C}(7)$ & $101.5(2)$ \\
\hline $\mathrm{C}(1)-\mathrm{Co}(1)-\mathrm{C}(7)$ & 101.9(2) \\
\hline $\mathrm{C}(3)-\mathrm{Co}(1)-\mathrm{Co}(2)$ & $79.85(18)$ \\
\hline $\mathrm{C}(2)-\mathrm{Co}(1)-\mathrm{Co}(2)$ & $85.74(16)$ \\
\hline
\end{tabular}




\begin{tabular}{|c|c|}
\hline $\mathrm{C}(1)-\mathrm{Co}(1)-\mathrm{Co}(2)$ & $84.96(16)$ \\
\hline $\mathrm{C}(7)-\operatorname{Co}(1)-\operatorname{Co}(2)$ & $167.35(15)$ \\
\hline$C(28)-P(1)-C(40)$ & $102.8(3)$ \\
\hline $\mathrm{C}(28)-\mathrm{P}(1)-\mathrm{C}(34)$ & $104.0(2)$ \\
\hline $\mathrm{C}(40)-\mathrm{P}(1)-\mathrm{C}(34)$ & $104.9(2)$ \\
\hline $\mathrm{C}(28)-\mathrm{P}(1)-\mathrm{Co}(2)$ & $112.31(17)$ \\
\hline $\mathrm{C}(40)-\mathrm{P}(1)-\mathrm{Co}(2)$ & $117.65(17)$ \\
\hline $\mathrm{C}(34)-\mathrm{P}(1)-\mathrm{Co}(2)$ & $113.65(18)$ \\
\hline$C(7)-N(1)-C(10)$ & $126.5(5)$ \\
\hline $\mathrm{C}(7)-\mathrm{N}(1)-\mathrm{C}(9)$ & $114.1(4)$ \\
\hline $\mathrm{C}(10)-\mathrm{N}(1)-\mathrm{C}(9)$ & $118.9(4)$ \\
\hline $\mathrm{O}(1)-\mathrm{C}(1)-\mathrm{Co}(1)$ & $174.1(5)$ \\
\hline $\mathrm{C}(5)-\mathrm{Co}(2)-\mathrm{C}(6)$ & $119.7(2)$ \\
\hline $\mathrm{C}(5)-\mathrm{Co}(2)-\mathrm{C}(4)$ & $114.7(3)$ \\
\hline $\mathrm{C}(6)-\mathrm{Co}(2)-\mathrm{C}(4)$ & $123.3(3)$ \\
\hline $\mathrm{C}(5)-\mathrm{Co}(2)-\mathrm{P}(1)$ & $98.52(18)$ \\
\hline $\mathrm{C}(6)-\mathrm{Co}(2)-\mathrm{P}(1)$ & $95.40(16)$ \\
\hline $\mathrm{C}(4)-\mathrm{Co}(2)-\mathrm{P}(1)$ & $91.44(17)$ \\
\hline $\mathrm{C}(5)-\mathrm{Co}(2)-\mathrm{Co}(1)$ & $89.40(18)$ \\
\hline $\mathrm{C}(6)-\mathrm{Co}(2)-\mathrm{Co}(1)$ & $82.35(16)$ \\
\hline $\mathrm{C}(4)-\mathrm{Co}(2)-\mathrm{Co}(1)$ & $83.32(17)$ \\
\hline $\mathrm{P}(1)-\mathrm{Co}(2)-\mathrm{Co}(1)$ & $171.81(5)$ \\
\hline $\mathrm{C}(7)-\mathrm{N}(2)-\mathrm{C}(19)$ & $127.7(4)$ \\
\hline $\mathrm{C}(7)-\mathrm{N}(2)-\mathrm{C}(8)$ & $113.6(4)$ \\
\hline $\mathrm{C}(19)-\mathrm{N}(2)-\mathrm{C}(8)$ & $117.8(4)$ \\
\hline $\mathrm{O}(2)-\mathrm{C}(2)-\mathrm{Co}(1)$ & $174.2(5)$ \\
\hline $\mathrm{O}(3)-\mathrm{C}(3)-\mathrm{Co}(1)$ & $178.1(5)$ \\
\hline $\mathrm{O}(4)-\mathrm{C}(4)-\mathrm{Co}(2)$ & $178.9(5)$ \\
\hline $\mathrm{O}(5)-\mathrm{C}(5)-\mathrm{Co}(2)$ & $178.3(5)$ \\
\hline $\mathrm{O}(6)-\mathrm{C}(6)-\mathrm{Co}(2)$ & $177.5(5)$ \\
\hline $\mathrm{N}(1)-\mathrm{C}(7)-\mathrm{N}(2)$ & $106.6(4)$ \\
\hline $\mathrm{N}(1)-\mathrm{C}(7)-\mathrm{Co}(1)$ & $126.9(4)$ \\
\hline $\mathrm{N}(2)-\mathrm{C}(7)-\mathrm{Co}(1)$ & $125.8(4)$ \\
\hline $\mathrm{N}(2)-\mathrm{C}(8)-\mathrm{C}(9)$ & $102.5(4)$ \\
\hline $\mathrm{N}(1)-\mathrm{C}(9)-\mathrm{C}(8)$ & $102.6(4)$ \\
\hline$C(15)-C(10)-C(11)$ & $121.9(5)$ \\
\hline
\end{tabular}




\begin{tabular}{|c|c|}
\hline$C(15)-C(10)-N(1)$ & $118.3(5)$ \\
\hline $\mathrm{C}(11)-\mathrm{C}(10)-\mathrm{N}(1)$ & $119.7(5)$ \\
\hline$C(12)-C(11)-C(10)$ & $117.4(5)$ \\
\hline $\mathrm{C}(12)-\mathrm{C}(11)-\mathrm{C}(16)$ & $120.5(5)$ \\
\hline$C(10)-C(11)-C(16)$ & $122.0(5)$ \\
\hline$C(11)-C(12)-C(13)$ & $122.1(5)$ \\
\hline$C(12)-C(13)-C(14)$ & $119.2(5)$ \\
\hline$C(12)-C(13)-C(17)$ & $120.9(5)$ \\
\hline$C(14)-C(13)-C(17)$ & $120.0(5)$ \\
\hline$C(15)-C(14)-C(13)$ & $120.5(5)$ \\
\hline$C(10)-C(15)-C(14)$ & $118.9(5)$ \\
\hline $\mathrm{C}(10)-\mathrm{C}(15)-\mathrm{C}(18)$ & $121.6(5)$ \\
\hline$C(14)-C(15)-C(18)$ & $119.5(5)$ \\
\hline$C(24)-C(19)-C(20)$ & $121.0(5)$ \\
\hline $\mathrm{C}(24)-\mathrm{C}(19)-\mathrm{N}(2)$ & $120.4(5)$ \\
\hline $\mathrm{C}(20)-\mathrm{C}(19)-\mathrm{N}(2)$ & $118.3(5)$ \\
\hline$C(21)-C(20)-C(19)$ & $117.2(5)$ \\
\hline$C(21)-C(20)-C(25)$ & $121.0(5)$ \\
\hline$C(19)-C(20)-C(25)$ & $121.8(5)$ \\
\hline$C(22)-C(21)-C(20)$ & $123.6(5)$ \\
\hline $\mathrm{C}(21)-\mathrm{C}(22)-\mathrm{C}(23)$ & $117.0(5)$ \\
\hline $\mathrm{C}(21)-\mathrm{C}(22)-\mathrm{C}(26)$ & $121.0(5)$ \\
\hline $\mathrm{C}(23)-\mathrm{C}(22)-\mathrm{C}(26)$ & $122.0(5)$ \\
\hline $\mathrm{C}(24)-\mathrm{C}(23)-\mathrm{C}(22)$ & $122.5(5)$ \\
\hline$C(23)-C(24)-C(19)$ & $118.7(5)$ \\
\hline $\mathrm{C}(23)-\mathrm{C}(24)-\mathrm{C}(27)$ & $119.8(5)$ \\
\hline$C(19)-C(24)-C(27)$ & $121.4(5)$ \\
\hline $\mathrm{C}(29)-\mathrm{C}(28)-\mathrm{C}(33)$ & $119.0(5)$ \\
\hline $\mathrm{C}(29)-\mathrm{C}(28)-\mathrm{P}(1)$ & $120.9(4)$ \\
\hline $\mathrm{C}(33)-\mathrm{C}(28)-\mathrm{P}(1)$ & $119.8(4)$ \\
\hline $\mathrm{C}(30)-\mathrm{C}(29)-\mathrm{C}(28)$ & $120.5(5)$ \\
\hline $\mathrm{C}(29)-\mathrm{C}(30)-\mathrm{C}(31)$ & $121.0(6)$ \\
\hline $\mathrm{C}(30)-\mathrm{C}(31)-\mathrm{C}(32)$ & $119.0(5)$ \\
\hline $\mathrm{C}(33)-\mathrm{C}(32)-\mathrm{C}(31)$ & $120.6(5)$ \\
\hline$C(32)-C(33)-C(28)$ & $119.9(6)$ \\
\hline $\mathrm{C}(35)-\mathrm{C}(34)-\mathrm{C}(39)$ & $117.9(5)$ \\
\hline
\end{tabular}




$\begin{array}{ll}\mathrm{C}(35)-\mathrm{C}(34)-\mathrm{P}(1) & 120.6(4) \\ \mathrm{C}(39)-\mathrm{C}(34)-\mathrm{P}(1) & 121.6(4) \\ \mathrm{C}(34)-\mathrm{C}(35)-\mathrm{C}(36) & 121.3(5) \\ \mathrm{C}(37)-\mathrm{C}(36)-\mathrm{C}(35) & 120.4(6) \\ \mathrm{C}(38)-\mathrm{C}(37)-\mathrm{C}(36) & 119.2(6) \\ \mathrm{C}(37)-\mathrm{C}(38)-\mathrm{C}(39) & 120.8(5) \\ \mathrm{C}(38)-\mathrm{C}(39)-\mathrm{C}(34) & 120.4(6) \\ \mathrm{C}(45)-\mathrm{C}(40)-\mathrm{C}(41) & 117.0(5) \\ \mathrm{C}(45)-\mathrm{C}(40)-\mathrm{P}(1) & 123.0(4) \\ \mathrm{C}(41)-\mathrm{C}(40)-\mathrm{P}(1) & 120.0(4) \\ \mathrm{C}(42)-\mathrm{C}(41)-\mathrm{C}(40) & 120.5(5) \\ \mathrm{C}(43)-\mathrm{C}(42)-\mathrm{C}(41) & 120.5(6) \\ \mathrm{C}(44)-\mathrm{C}(43)-\mathrm{C}(42) & 119.8(6) \\ \mathrm{C}(43)-\mathrm{C}(44)-\mathrm{C}(45) & 120.5(6) \\ \mathrm{C}(44)-\mathrm{C}(45)-\mathrm{C}(40) & 121.8(6)\end{array}$

Symmetry transformations used to generate equivalent atoms: 
Table 9. Anisotropic displacement parameters $\left(\AA^{2} \times 10^{3}\right)$ for 9. The anisotropic displacement factor exponent takes the form: $-2 \pi^{2}\left[h^{2} a^{* 2} U^{11}+\ldots+2 h k a^{*} b^{*} U^{12}\right]$

\begin{tabular}{|c|c|c|c|c|c|c|}
\hline & $\mathrm{U}^{11}$ & $\mathrm{U}^{22}$ & $\mathrm{U}^{33}$ & $\mathrm{U}^{23}$ & $\mathrm{U}^{13}$ & $\mathrm{U}^{12}$ \\
\hline $\operatorname{Co}(1)$ & $17(1)$ & $18(1)$ & $16(1)$ & $-2(1)$ & $2(1)$ & $-2(1)$ \\
\hline $\mathrm{P}(1)$ & $18(1)$ & $19(1)$ & $17(1)$ & $0(1)$ & $0(1)$ & $-2(1)$ \\
\hline $\mathrm{O}(1)$ & $30(3)$ & $50(3)$ & $22(2)$ & $-5(2)$ & $-10(2)$ & $2(2)$ \\
\hline $\mathrm{N}(1)$ & $21(3)$ & $26(2)$ & $14(3)$ & $0(2)$ & $0(2)$ & $1(2)$ \\
\hline $\mathrm{C}(1)$ & 11(3) & $27(3)$ & $26(4)$ & $-4(3)$ & $7(3)$ & $0(2)$ \\
\hline $\mathrm{Co}(2)$ & $19(1)$ & $18(1)$ & $19(1)$ & $0(1)$ & $2(1)$ & $-2(1)$ \\
\hline $\mathrm{O}(2)$ & $29(3)$ & $31(2)$ & $40(3)$ & $10(2)$ & $13(2)$ & $-6(2)$ \\
\hline $\mathrm{N}(2)$ & $19(3)$ & $29(2)$ & $13(2)$ & $-1(2)$ & $1(2)$ & $-9(2)$ \\
\hline $\mathrm{C}(2)$ & $17(3)$ & $22(3)$ & $23(3)$ & $-8(2)$ & $-4(3)$ & $3(2)$ \\
\hline $\mathrm{O}(3)$ & $31(3)$ & $35(2)$ & $46(3)$ & $-5(2)$ & $-1(2)$ & $-12(2)$ \\
\hline $\mathrm{C}(3)$ & $19(3)$ & $22(3)$ & $24(3)$ & $-1(2)$ & $2(3)$ & $-8(3)$ \\
\hline $\mathrm{O}(4)$ & $40(3)$ & $32(2)$ & $35(3)$ & $13(2)$ & $11(2)$ & $-5(2)$ \\
\hline $\mathrm{C}(4)$ & $23(3)$ & $19(3)$ & $33(4)$ & $-5(3)$ & $0(3)$ & $-8(2)$ \\
\hline $\mathrm{O}(5)$ & $39(3)$ & $47(3)$ & $47(3)$ & $-1(2)$ & $-8(2)$ & $-20(2)$ \\
\hline $\mathrm{C}(5)$ & $22(3)$ & $26(3)$ & $24(3)$ & $3(2)$ & $1(3)$ & $-9(3)$ \\
\hline $\mathrm{O}(6)$ & $40(3)$ & $43(2)$ & $21(2)$ & $-1(2)$ & $-4(2)$ & $-4(2)$ \\
\hline$C(6)$ & $14(3)$ & $18(3)$ & $29(4)$ & $-8(2)$ & $11(3)$ & $3(2)$ \\
\hline$C(7)$ & $23(3)$ & $17(3)$ & $10(3)$ & $-5(2)$ & $1(2)$ & $-9(2)$ \\
\hline$C(8)$ & $22(3)$ & $32(3)$ & $24(3)$ & $4(3)$ & $8(3)$ & $6(3)$ \\
\hline$C(9)$ & $12(3)$ & $29(3)$ & $25(3)$ & $-3(2)$ & $2(2)$ & $-3(2)$ \\
\hline$C(10)$ & $18(3)$ & $31(3)$ & $7(3)$ & $-2(2)$ & $9(2)$ & $1(2)$ \\
\hline $\mathrm{C}(11)$ & $20(3)$ & $23(3)$ & $20(3)$ & $3(2)$ & $4(2)$ & $-7(2)$ \\
\hline$C(12)$ & $23(3)$ & $28(3)$ & $24(3)$ & $8(3)$ & $2(3)$ & $-7(3)$ \\
\hline$C(13)$ & $20(3)$ & $36(3)$ & $21(3)$ & $5(3)$ & $-2(3)$ & $-6(3)$ \\
\hline$C(14)$ & $15(3)$ & $33(3)$ & $26(3)$ & $-5(3)$ & $3(3)$ & $-8(2)$ \\
\hline$C(15)$ & $14(3)$ & $25(3)$ & $20(3)$ & $-4(2)$ & $5(2)$ & $2(2)$ \\
\hline$C(16)$ & $58(5)$ & $33(3)$ & $25(4)$ & $5(3)$ & $-1(3)$ & $-18(3)$ \\
\hline$C(17)$ & $32(4)$ & $54(4)$ & $26(4)$ & 4(3) & $-9(3)$ & $-15(3)$ \\
\hline$C(18)$ & $35(4)$ & $30(3)$ & $39(4)$ & $-2(3)$ & $-1(3)$ & $-11(3)$ \\
\hline$C(19)$ & $20(3)$ & $25(3)$ & $12(3)$ & $2(2)$ & $-1(2)$ & $0(2)$ \\
\hline $\mathrm{C}(20)$ & $19(3)$ & $18(3)$ & $18(3)$ & $0(2)$ & $-7(2)$ & $3(2)$ \\
\hline
\end{tabular}




\begin{tabular}{|c|c|c|c|c|c|c|}
\hline$C(21)$ & $22(3)$ & $27(3)$ & $24(3)$ & $6(3)$ & $-1(3)$ & $-4(3)$ \\
\hline$C(22)$ & $23(3)$ & $27(3)$ & $19(3)$ & $4(2)$ & $-3(3)$ & 1(3) \\
\hline$C(23)$ & $33(4)$ & $35(3)$ & $19(3)$ & $-3(3)$ & $-9(3)$ & $-3(3)$ \\
\hline$C(24)$ & 29(4) & $23(3)$ & $23(3)$ & $3(2)$ & $-11(3)$ & $-3(3)$ \\
\hline$C(25)$ & $30(4)$ & $23(3)$ & $26(3)$ & $-4(2)$ & $5(3)$ & $-9(3)$ \\
\hline$C(26)$ & $51(5)$ & $52(4)$ & $23(4)$ & $10(3)$ & $3(3)$ & $0(3)$ \\
\hline$C(27)$ & $57(5)$ & $47(4)$ & $39(4)$ & $1(3)$ & $-17(4)$ & $-32(4)$ \\
\hline$C(28)$ & $23(3)$ & 19(3) & $16(3)$ & $-4(2)$ & 1(2) & $3(2)$ \\
\hline$C(29)$ & $18(3)$ & $26(3)$ & $34(4)$ & $-1(3)$ & $-3(3)$ & $-11(3)$ \\
\hline$C(30)$ & $27(4)$ & $34(3)$ & $31(4)$ & $-7(3)$ & $-6(3)$ & $-10(3)$ \\
\hline$C(31)$ & $28(4)$ & $38(3)$ & $19(3)$ & $-4(3)$ & $-9(3)$ & $4(3)$ \\
\hline$C(32)$ & $22(3)$ & $38(3)$ & 19(3) & $6(3)$ & $1(3)$ & $-6(3)$ \\
\hline$C(33)$ & $17(3)$ & $26(3)$ & $20(3)$ & $1(2)$ & 1(2) & $0(2)$ \\
\hline$C(34)$ & $18(3)$ & $26(3)$ & 11(3) & $5(2)$ & $-1(2)$ & $-2(2)$ \\
\hline$C(35)$ & $22(3)$ & $25(3)$ & $23(3)$ & $6(2)$ & $-2(3)$ & $2(3)$ \\
\hline$C(36)$ & $28(4)$ & $31(3)$ & $29(4)$ & $4(3)$ & $-5(3)$ & $-14(3)$ \\
\hline$C(37)$ & $44(4)$ & $25(3)$ & $33(4)$ & $-2(3)$ & $-7(3)$ & $-12(3)$ \\
\hline$C(38)$ & $43(4)$ & $26(3)$ & $29(4)$ & $-5(3)$ & $-1(3)$ & $3(3)$ \\
\hline C(39) & $26(4)$ & 28(3) & $32(4)$ & $-3(3)$ & $-4(3)$ & $-6(3)$ \\
\hline$C(40)$ & $16(3)$ & $30(3)$ & $19(3)$ & $-1(2)$ & $-2(2)$ & $-8(2)$ \\
\hline $\mathrm{C}(41)$ & $29(4)$ & $28(3)$ & $30(4)$ & $-1(3)$ & $-4(3)$ & $-9(3)$ \\
\hline$C(42)$ & 41(4) & $42(4)$ & $24(4)$ & $3(3)$ & $3(3)$ & $-17(3)$ \\
\hline$C(43)$ & $30(4)$ & $55(4)$ & 21(3) & $-2(3)$ & $8(3)$ & $-24(3)$ \\
\hline$C(44)$ & $29(4)$ & $53(4)$ & 21(3) & $-2(3)$ & $7(3)$ & $-2(3)$ \\
\hline$C(45)$ & $24(4)$ & $37(3)$ & $29(4)$ & $2(3)$ & $2(3)$ & $-3(3)$ \\
\hline
\end{tabular}


Table 10. Hydrogen coordinates ( $\left.\mathrm{x} 10^{4}\right)$ and isotropic displacement parameters $\left(\AA^{2} \times 10^{3}\right)$ for 9 .

\begin{tabular}{|c|c|c|c|c|}
\hline & $\mathrm{x}$ & $\mathrm{y}$ & $\mathrm{z}$ & $\mathrm{U}(\mathrm{eq})$ \\
\hline $\mathrm{H}(8 \mathrm{~A})$ & 9991 & -1022 & 2019 & 35 \\
\hline $\mathrm{H}(8 \mathrm{~B})$ & 10628 & -23 & 2268 & 35 \\
\hline $\mathrm{H}(9 \mathrm{~A})$ & 10473 & 694 & 1150 & 27 \\
\hline $\mathrm{H}(9 \mathrm{~B})$ & 10044 & -386 & 881 & 27 \\
\hline $\mathrm{H}(12)$ & 7043 & 3269 & -590 & 30 \\
\hline $\mathrm{H}(14)$ & 6346 & 280 & -855 & 29 \\
\hline $\mathrm{H}(16 \mathrm{~A})$ & 8292 & 3663 & 391 & 57 \\
\hline $\mathrm{H}(16 \mathrm{~B})$ & 9022 & 2702 & 934 & 57 \\
\hline $\mathrm{H}(16 \mathrm{C})$ & 7291 & 3401 & 1081 & 57 \\
\hline $\mathrm{H}(17 \mathrm{~A})$ & 6775 & 1692 & -1952 & 54 \\
\hline $\mathrm{H}(17 \mathrm{~B})$ & 6046 & 2929 & -1667 & 54 \\
\hline $\mathrm{H}(17 \mathrm{C})$ & 5064 & 2058 & -1603 & 54 \\
\hline $\mathrm{H}(18 \mathrm{~A})$ & 6625 & -1007 & 84 & 51 \\
\hline $\mathrm{H}(18 \mathrm{~B})$ & 6784 & -624 & 888 & 51 \\
\hline $\mathrm{H}(18 \mathrm{C})$ & 8268 & -1130 & 360 & 51 \\
\hline $\mathrm{H}(21)$ & 6523 & -1671 & 3991 & 30 \\
\hline $\mathrm{H}(23)$ & 8208 & 674 & 4752 & 36 \\
\hline $\mathrm{H}(25 \mathrm{~A})$ & 6225 & -1856 & 2747 & 40 \\
\hline $\mathrm{H}(25 \mathrm{~B})$ & 7864 & -1923 & 2359 & 40 \\
\hline $\mathrm{H}(25 \mathrm{C})$ & 6447 & -910 & 2201 & 40 \\
\hline $\mathrm{H}(26 \mathrm{~A})$ & 5895 & -836 & 5347 & 67 \\
\hline $\mathrm{H}(26 \mathrm{~B})$ & 7299 & -454 & 5602 & 67 \\
\hline $\mathrm{H}(26 \mathrm{C})$ & 7546 & -1686 & 5316 & 67 \\
\hline $\mathrm{H}(27 \mathrm{~A})$ & 10085 & 1288 & 3709 & 65 \\
\hline $\mathrm{H}(27 \mathrm{~B})$ & 8479 & 2191 & 3810 & 65 \\
\hline $\mathrm{H}(27 \mathrm{C})$ & 9135 & 1700 & 3021 & 65 \\
\hline $\mathrm{H}(29)$ & -1682 & 4868 & 2447 & 30 \\
\hline $\mathrm{H}(30)$ & -3101 & 5513 & 1476 & 36 \\
\hline $\mathrm{H}(31)$ & -2347 & 6681 & 626 & 37 \\
\hline $\mathrm{H}(32)$ & -136 & 7225 & 773 & 32 \\
\hline
\end{tabular}




$\begin{array}{lrlll}\mathrm{H}(33) & 1298 & 6598 & 1755 & 27 \\ \mathrm{H}(35) & 4145 & 5315 & 3119 & 30 \\ \mathrm{H}(36) & 5125 & 6718 & 3485 & 34 \\ \mathrm{H}(37) & 3521 & 8463 & 3768 & 40 \\ \mathrm{H}(38) & 931 & 8780 & 3706 & 42 \\ \mathrm{H}(39) & -77 & 7379 & 3341 & 34 \\ \mathrm{H}(41) & 1033 & 3390 & 3978 & 34 \\ \mathrm{H}(42) & -647 & 3212 & 4956 & 42 \\ \mathrm{H}(43) & -2823 & 4626 & 5252 & 40 \\ \mathrm{H}(44) & -3349 & 6215 & 4565 & 44 \\ \mathrm{H}(45) & -1735 & 6402 & 3581 & 38 \\ \end{array}$

